\title{
Spin and Charge Current in a Short Semiconducting Chain of Paramagnetic Ionic Blocks
}

\author{
A. Lehmann-Szweykowska, R.J. Wojciechowski and R. Micnas \\ Faculty of Physics, Adam Mickiewicz University \\ Umultowska 85, 61-614 Poznań, Poland
}

\begin{abstract}
We deal with the electric current flowing through a short chain of paramagnetic ionic blocks, coupled to metallic electrodes in the serial configuration. An original three-band Hubbard-Anderson Hamiltonian is diagonalised at the level of the single ionic block. A minimal but sufficient set of the latter's four hybridised eigenstates serves as a basis for the determination of the time-ordered temperature-dependent matrix Green functions, in terms of which all the current-voltage $(I-V)$ characteristics can be expressed provided the coupling to the electrodes is weak. The separation of the opposite-spin contributions to the electric current and, consequently, the spin current from the left to right electrode can result from the on-site Coulomb repulsion term of Hubbard-Anderson Hamiltonian, with no spin polarisation at the electrodes, but with the Zeeman-like coupling of the centre to either a molecular or an external magnetic field.
\end{abstract}

PACS numbers: 72.25.Dc, 73.61.Le, 73.63.Rt

\section{Introduction}

In a discussion of the proposed microscopic model of spin and charge transport in semiconducting materials with permanent magnetic moments, we concentrate on two non-trivial aspects of the problem, which are, first, a specific treatment of the on-site Coulomb repulsion in the three-band Hubbard-Anderson $(\mathrm{H}-\mathrm{A})$ model and the resulting separation of the opposite-spin contributions to the electric current; and, second, the influence of molecular and external magnetic fields on the energy spectrum of a single ionic block. The method, employed throughout this paper $[1,2]$, is designed for such systems, whose natural building units are ionic clusters. This is true about many semiconducting compounds with permanent magnetic moments, but especially about the transition metal oxides and/or manganites. The ionic blocks consist of the central paramagnetic cations, such as transition or rare-earth ions, surrounded by the oxygen anions. The neighbouring blocks share one (or more) anion sites. For instance, in yttrium-iron garnet (YIG), they are tetrahedrons with the central iron site, surrounded by four oxygen ions, octahedrons in which the central iron sites have six oxygen neighbours, and dodecahedrons with the central yttrium ions, each one of which is with eight oxygen ions at the corners. In manganites, the oxygen ions are located at the corners of octahedrons with the cations at their centres.

The main objective of this paper is to compute the charge and spin currents through a minimal but sufficiently long chain, which consists of three ionic blocks. A point symmetry of the outer blocks is different from that of the middle one $[3,4]$. The outer blocks are coupled to non-interacting metallic electrodes in a serial configuration. As shown earlier, an extra charge, localised temporarily at one of the outer blocks, can induce a change of the exchange interaction between the spins of the outer and middle clusters from the Anderson antiferromagnetic to ferromagnetic [1]. This means that the chain becomes actually asymmetric. The charge and spin currents, from the left and the right electrodes, can be defined, respectively, as $I_{Q(S)}=\frac{1}{2}\left(I_{\left(m_{\mathrm{s}}\right)} \pm I_{\left(-m_{\mathrm{s}}\right)}\right)$. By the non-equilibrium Green function method [5], due to weak coupling between the centre and the electrodes, one can obtain a well-known Meir-Wingreen formula [6] for the $m_{\mathrm{s}}$-current from the left electrode $[5,7]$ :

$$
\begin{aligned}
& I_{m_{\mathrm{s}}}=\mathrm{i} q(2 h)^{-1} \operatorname{Tr} \int \mathrm{d} E \Gamma_{L, m_{\mathrm{s}}} \\
& \quad \times\left[G_{L, m_{\mathrm{s}}}^{<}(E)+f_{L}(E)\left(G_{L, m_{\mathrm{s}}}^{\mathrm{r}}(E)-G_{L, m_{\mathrm{s}}}^{\mathrm{a}}(E)\right)\right] .(1)
\end{aligned}
$$
$G_{L, m_{\mathrm{s}}}^{\mathrm{r}}(E), G_{L, m_{\mathrm{s}}}^{\mathrm{a}}(E)$ and $G_{L, m_{\mathrm{s}}}^{<}(E)$ are the matrix retarded, advanced and lesser Green functions, respectively, which are constructed from the fermion operators, defined in a basis of the hybridised eigenstates of the first ionic block, coupled to the left electrode. $\Gamma_{L, m_{\mathrm{s}}}$ is the matrix line-width function and $f_{L}(E)$ is the Fermi-Dirac distribution function for the left electrode.

\section{Cluster energy structure and the current}

The computation of the current requires finding single-cluster eigenstates with the appropriate energy levels. The first task is then a diagonalisation of the threeband Hubbard Hamiltonian, projected onto a single cluster, with a two-site hybridisation $\hat{H}_{p d}$ between the $d(f)$ 
atomic states of the central cations and $p$ or $s$ states of the anions, considered a perturbation, while temporarily neglecting a possible inter-block hybridisation. The on-site Coulomb repulsion at the cation sites is restricted to its maximal part between opposite-spin electrons. For our purpose, it turns out to be sufficient to consider fourdimensional Hilbert space per cluster. The original basis consists of two single-electron $d$ states at the cation with mutually opposite spins and energies equal to $E_{d}$ and $E_{d}+U$, respectively, and two $2 p$ states with opposite spins and the same energy $E_{p}$ which are localised with equal probability at all of the anions of the cluster. As a result of the diagonalisation with respect to the intra-block hybridisation, four "active" single-fermion eigenstates of each block $\left(\Psi_{\mu_{i}, m_{\mathrm{s}}}\right)$ are obtained in the form of linear combinations of the originally selected states. Coefficients of the wave functions explicitly depend upon the Hubbard parameter, $U$ and so do the corresponding energy levels. For the states with the spin $m_{\mathrm{s}}$ one obtains $E_{\mu_{i}, m_{\mathrm{s}}}=\frac{1}{2}\left(E_{d}+E_{p}\right) \pm \frac{1}{2} \sqrt{\left(E_{d}-E_{p}\right)^{2}+4 V_{p d}^{2}}$, whereas for those with the opposite spins one gets $E_{\mu_{i},-m_{\mathrm{s}}}=$ $\frac{1}{2}\left(E_{d}+U+E_{p}\right) \pm \frac{1}{2} \sqrt{\left(E_{d}+U-E_{p}\right)^{2}+4 V_{p d}^{2}} \cdot i=1,2,3$ labels the ionic blocks and $\mu=1,2,3,4$ labels their respective eigenstates [1-3]. In our convention, $m_{\mathrm{s}}$ is the spin number projection, attached to the states $\mu=1,3$, whereas $-m_{\mathrm{s}}$ is the spin number projection of $\mu=2,4$. In the new basis, consisting of the four hybridised eigenstates per a block, creation $\left(\hat{\Psi}_{\mu_{i}}^{+}\right)$and annihilation $\left(\hat{\Psi}_{\mu_{i}}\right)$ fermion operators are defined (cf. [3-5]). Consequently, the truncated Hamiltonian of the chain coupled to the metallic electrodes can be rewritten in a simple quadratic form [1]:

$$
\begin{aligned}
\hat{H}_{\mathrm{c}} & =\sum_{\mu_{i}} E_{\mu_{i}} \hat{\Psi}_{\mu_{i}}^{+} \hat{\Psi}_{\mu_{i}}+\sum_{\mu_{i}, \nu_{j}}\left(V_{p d}\left(\mu_{i}, \nu_{j}\right) \hat{\Psi}_{\mu_{i}}^{+} \hat{\Psi}_{\nu_{j}}+\text { h.c. }\right) \\
& +\sum_{\mu_{i}, \boldsymbol{k}, m_{\mathrm{s}}^{\prime}}\left(V_{\mu_{i}}\left(\boldsymbol{k}, m_{\mathrm{s}}^{\prime}\right) \hat{\Psi}_{\mu_{i}}^{+} \hat{c}_{\boldsymbol{k}, m_{\mathrm{s}}^{\prime}}+\text { h.c. }\right),
\end{aligned}
$$

where $V_{p d}\left(\mu_{i}, \nu_{j}\right)=\left\langle\Psi_{\nu_{j}}\left|\hat{H}_{p d}\right| \Psi_{\mu_{i}}\right\rangle \delta_{m_{\mathrm{s}} m_{\mathrm{s}}^{\prime}}$ and analogously, $V_{\mu_{i}}\left(\boldsymbol{k}, m_{\mathrm{s}}^{\prime}\right)=\left\langle\phi_{\boldsymbol{k}, m_{\mathrm{s}}^{\prime}}\left|\hat{H}_{T}\right| \Psi_{\mu_{i}, m_{\mathrm{s}}}\right\rangle \delta_{m_{\mathrm{s}} m_{\mathrm{s}}^{\prime}} . \quad \hat{c}_{\boldsymbol{k}, m_{\mathrm{s}}^{\prime}}^{+}$ and $\hat{c}_{\boldsymbol{k}, m_{\mathrm{s}}^{\prime}}$ are the second quantisation operators for electrodes. As seen, no spin flipping processes are induced. An external magnetic and/or molecular field $(B)$ can significantly change the mutual positions of the cluster's energy levels. Shifts of the energy levels can be computed in the first order of the perturbation theory, considering the Zeeman term as a perturbation, with possible orbital contribution to the magnetic moment [2].

In frequency space, matrix elements of the $2 \times 2$ matrix Green functions $G^{\mathrm{r}}$, which are needed for the computation of the left spin and/or charge current, are $\left\langle\left\langle\hat{\Psi}_{\mu_{1}}^{+} ; \hat{\Psi}_{\mu_{1}^{\prime}}\right\rangle\right\rangle_{\omega}^{\mathrm{r}}$, where a short-hand (Zubarev) notation is introduced. For $m_{\mathrm{s}}$-contribution to the current $\mu_{1}, \mu_{1}^{\prime}=$ 1,3 , whereas for $-m_{\mathrm{s}}$-contribution $\mu_{1}, \mu_{1}^{\prime}=2,4$. The anticommutator retarded Green functions can be derived with the equation-of-motion technique [3-5]. The method yields two separate sets, each of six linear equa- tions, for two spin orientations. These equations can be solved numerically, however, formulae for the retarded Green functions can be also written explicitly. Matrix elements of the $2 \times 2$ matrix line-width functions $\Gamma_{L, m_{\mathrm{s}}}$ are proportional to $V_{\mu_{1}}\left(\boldsymbol{k}, m_{\mathrm{s}}\right) V_{\mu_{1}^{\prime}}^{*}\left(\boldsymbol{k}, m_{\mathrm{s}}\right)$, where again $\mu_{1}, \mu_{1}^{\prime}=1,3$ refers to the "up-spin" current and $\mu_{1}, \mu_{1}^{\prime}=2,4$ for the "down-spin" current. Since the attention is to be focused on a crucial role of the Coulomb on-site interaction and also, on the new way of treating it, proposed in the paper, a more detailed discussion of the line-width function will be given elsewhere. Here, the line-width matrix elements $\left(V_{\mu_{i}}\left(\boldsymbol{k}, m_{\mathrm{s}}^{\prime}\right)\right)$ are simply parameterised and put equal to one another $\left(V_{T}\right)$.

\section{Results and discussion}

The spin current-voltage characteristics for different values of the Hubbard parameter, $U$, both without and with the external magnetic field are presented in Fig. 1. The current is computed at low temperature. The diagrams in Fig. 1 clearly prove validity of the thesis that $U$ can be responsible for separation of the opposite-spin contributions to the current. The effect, however, is bet-

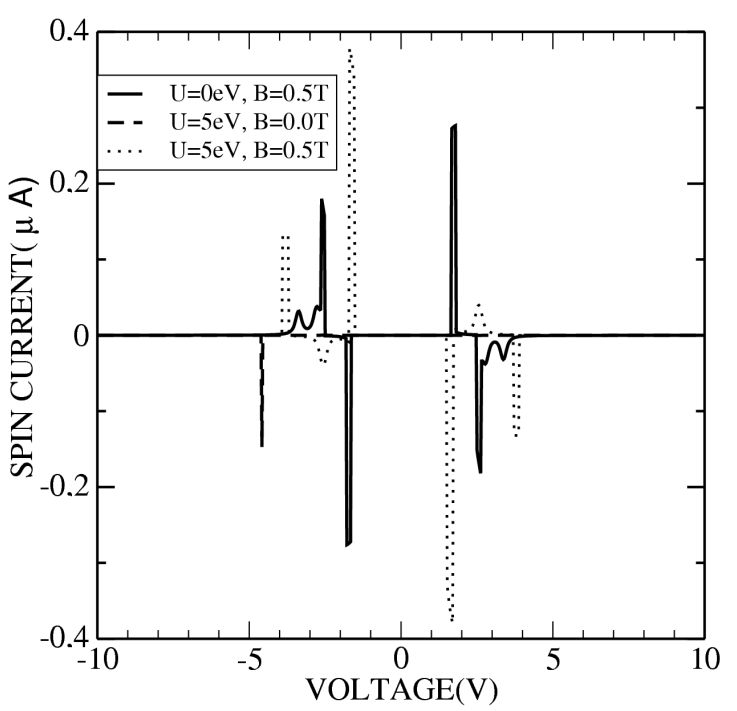

Fig. 1. The spin current vs. voltage for different values of $U$ and $B$ and temperature $T=10 \mathrm{~K}$.

ter visible in a presence of the magnetic field, which singles out the quantisation axis, breaking an invariance of the Hamiltonian with the respect to the exchange of the spin indexes (see the curve $U=5 \mathrm{eV}$ and $B=0$ in Fig. 1 and the analogous curve in Fig. 2). The resonant-like form of the curves in Fig. 1, reflects the energy structure of the cluster with the peaks, corresponding to transitions between its energy levels, connected with the appropriate spin flips. For $U=0$ and $B=0$ the spin current must vanish because each cluster has only two hybridised spin-degenerated energy levels, the charge current, however, occurs in the system (Fig. 2). As shown in Fig. 2, the 


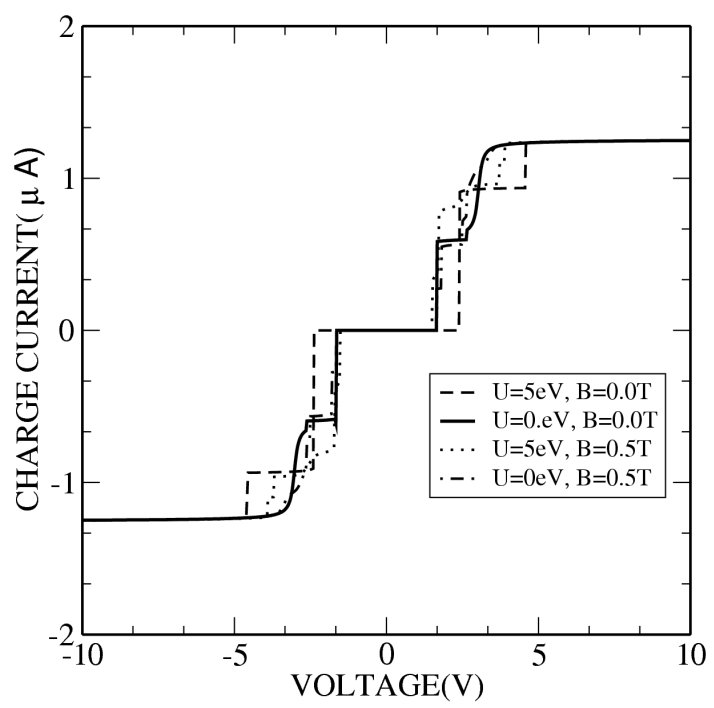

Fig. 2. The charge current vs. voltage for different values of $U$ and $B$ and temperature $T=10 \mathrm{~K}$.

influence of $U$ on the charge current is much less pronounced than it is in the case of the spin current and their step-like form reflects nicely the energy structure of the ionic clusters. All the curves actually represent not the net current, but only its one (left) branch.

To close the discussion let us briefly summarise the main conclusions. The proposed simple model can be applied to any system, whose building units are ionic blocks. The original $\mathrm{H}-\mathrm{A}$ Hamiltonian, projected onto a single ionic cluster, is diagonalised by linear combination of molecular orbitals (LCMO) method. The minimal but sufficient set of four active eigenstates per clus- ter is established to provide the convenient basis for the further procedure. The separation of the opposite-spin eigenstates, due to the on-site Coulomb repulsion at the cations, included in the formalism, gives rise to the different opposite-spin currents, without spin polarisation at the electrodes. The interaction with the field is taken into account by the appropriate Zeeman term in the original Hamiltonian. The influence of either molecular or an external magnetic field both emphasises and enriches the effect. The Meir-Wingreen formulae for the spin and charge currents are computed in terms of the matrix retarded Green functions, defined in the basis of the single-cluster active eigenstates. The occurrence of the spin current is favoured in ferromagnetic semiconductors and/or with magnetically-ordered electrodes.

\section{References}

[1] R.J. Wojciechowski, A. Lehmann-Szweykowska, R. Micnas, G.A. Gehring, P.E. Wigen, Phys. Rev. B 69, 2144341 (2004).

[2] A. Lehmann-Szweykowska, R.J. Wojciechowski, G.A. Gehring, A. Szamer, P.E. Wigen, R. Micnas, Phys. Status Solidi B 244, 2487 (2007).

[3] A. Lehmann-Szweykowska, R.J. Wojciechowski, R. Micnas, J. Phys., Conf. Series 104, 012024 (2008).

[4] T. Kostyrko, B. Bułka, Mater. Sci. 22, 497 (2004).

[5] H. Haug, A.-P. Jauho, Quantum Kinetics in Transport and Optics of Semiconductors, Springer-Verlag, Berlin 1998.

[6] Y. Meir, N.S. Wingreen, Phys. Rev. Lett. 68, 2512 (1992).

[7] T. Kostyrko, B. Bułka, Phys. Rev. B 71, 235306 (2005). 